\title{
Energy Harvesting - Power Supplies without Batteries or Cables
}

\author{
Peter Spies \\ Group Manager Integrated Energy Supplies \\ Fraunhofer IIS \\ Nordostpark 93 \\ 90411 Nuremberg \\ Germany
}

Energy Harvesting makes use of minimum amounts of ambient energy like thermal gradients, vibrations or light. Collecting these amounts of energy a long time to power short actions like sensor measurements or wireless data transmissions is the key of energy harvesting. A lot of research and development activities are on-going world-wide, addressing materials, devices, systems and applications. Promising field trials are carried out in various application areas, most of them in wireless sensor networks. First products are available, mainly in the industrial and automation area whereas consumer products are predicted for the next ten years. The characterization of potential ambient energy sources shows a broad range of the typical parameters and thus big challenges for the system design. Different development approaches are possible to arrive at a cost-optimized power supply solution. Generic energy harvesting systems are composed of an energy transducer, a power management, some kind of energy storage and the final application. Matching of the different functional blocks will result in maximum energy output, minimum device cost and board space.

\section{Overview and Architecture}

Energy Harvesting makes use of minimum amounts of ambient energy like thermal gradients, vibrations or light. Collecting and storing these amounts of energy for a long time to power short actions like sensor measurements or wireless data transmissions is the key of energy harvesting. Thus, in a generic approach, an energy harvesting system must have some kind of energy storage device. This might be a battery or a capacitor, depending on application, environment, costs, available board space and allowed leakage currents. Furthermore, most electronic systems like wireless transceivers or micro-processors work in burst mode or time-division mode (FDMA, FDD). Thus, pulse currents to power typical transmission bursts are required. These pulse currents cannot be provided by state-of-the-art energy harvesting transducers like solar cells, thermo-generators or piezotransducers due to their internal resistance. This is another reason to incorporate small or medium storage elements in these power supplies.

Energy transducers do not provide the appropriate voltage level to charge a battery or a capacitor. Their output voltage level depends on their design and the amount of available ambient energy in terms of vibration amplitude, thermal gradient or light intensity. Thus, some kind of interfacing electronics in form of a power management circuit like dc-dc or ac-dc regulator is required to take full use of the electrical energy delivered by the transducer. According to power theory, the maximum power from a source is only provided to a matched load. For that reason, the duty of the power management is also matching as precise as possible to the internal resistance of the transducer. This is done in large solar plants with the help of maximum power point trackers (MPPT) and should be adapted to energy harvesting in some kind to achieve the most power from the transducer. MPPT is carried out by sensing the output power and controlling the input current of the voltage regulator. The challenge is to realize this additional functionality very power efficient without degrading the overall efficiency of the energy harvesting system. MPPT is especially useful in applications with a large 'power dynamic' which can be significant fluctuations in thermal gradient or large changes in vibration amplitude. On the other hand, in a system with constant power, fixing the input current to a certain level might be sufficient and more power efficient. 


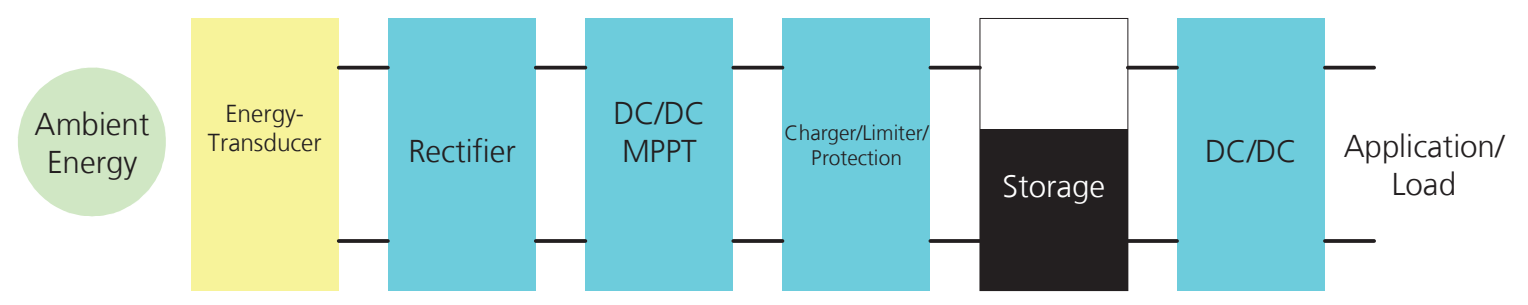

Picture 1: Generic architecture of energy harvesting power supply

\section{Applications and first installations}

The first applications and successful installations of energy harvesting were those where high device costs are not a problem and where large device dimensions are acceptable. This is often the case when expensive equipment is monitored or when the detection of a malfunction can save much money and avoid the down-time of a plant or technical system. Typical examples are:

\section{Condition Monitoring}

An early detection of an abrasion or fault and appropriate maintenance in a technical system can avoid a total down-time or even worse consequences and in most cases save a lot of money. In those cases, the installation costs of a wireless sensor with an energy harvesting, maintenance-free power supply is much lower than the costs for final repair or overhaul or the expenses when the whole system breaks down.

Typical measurement parameters in this application are the vibration frequencies of systems like pumps, engines, turbines, etc. With the help of these measurements, the state-of-health can be evaluated. If significant changes from the typical vibration frequencies are detected, it is obvious that a technical problem, like an imbalance is approaching. These vibrations can also be used for energy harvesting, whereas changes from the typical ranges have to be considered in the system design to avoid running out of power in fault occurrence. Here, broadband energy harvesting systems are required, which use a wider spectrum of frequencies for power supply [1].

\section{Structural Health Monitoring}

The principle of monitoring expensive goods is also valid in structural health monitoring. Buildings like bridges and skyscrapers but also large vehicles like planes or ships are difficult to monitor in their whole dimensions. Several tens or hundreds of individual test points are required and sometimes only the real-time combination and analysis of all the measurement data enables a serious investigation of the whole structure. Wireless sensor networks are the solution to that problem, leaving another problem of avoiding tens or hundreds of batteries to energy harvesting. Especially in large systems like buildings or huge vehicles, the vibrations frequencies are low due to their high weight and dimensions. Furthermore, vibrations in these applications are usually unwanted, driving the engineers to take measures to suppress them. The consequences in these applications are only very small vibrations in the range of $0.1 \mathrm{~g}$ or below and low frequencies, making it difficult to harvest enough power for the sensor or transceiver modules. Picture 2 shows the vibration measurements in a vehicle driving inner-city. The advantages of huge dimensional applications are that large and expensive transducers are acceptable, which increase their output power by combining several transducers and adding their output currents.

\section{Building automation}

In large buildings, the advantage of avoiding cables for power supply is the main reason for the employment of energy harvesting. Especially in retrofit equipment, but also to reduce wiring costs and labour time, energy harvesting is already used in numberless installations. These are often light switching system, heating controls, anti-theft protection systems etc. [2]. 


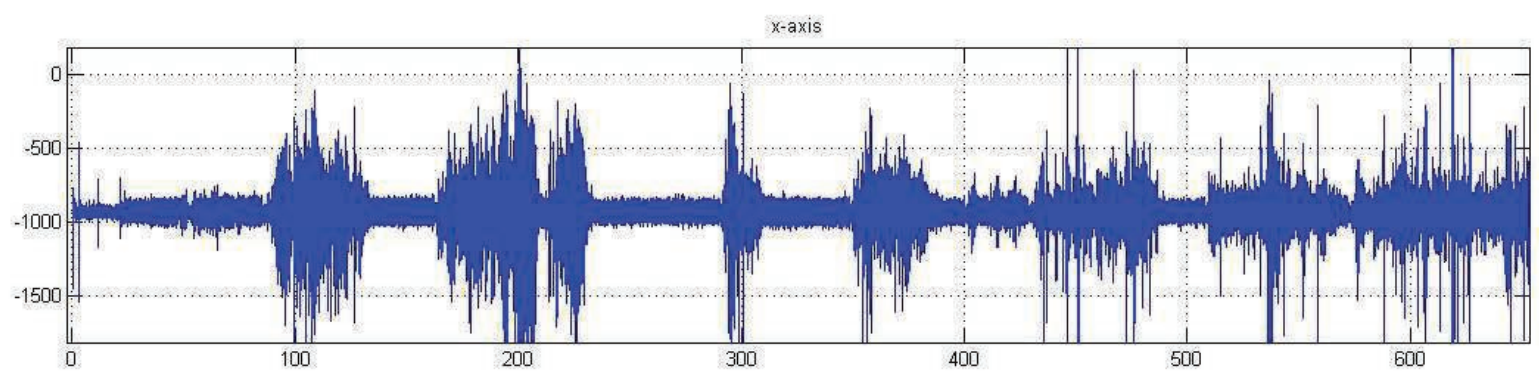

Single-Sided Amplitude Spectrum of $x(t)$

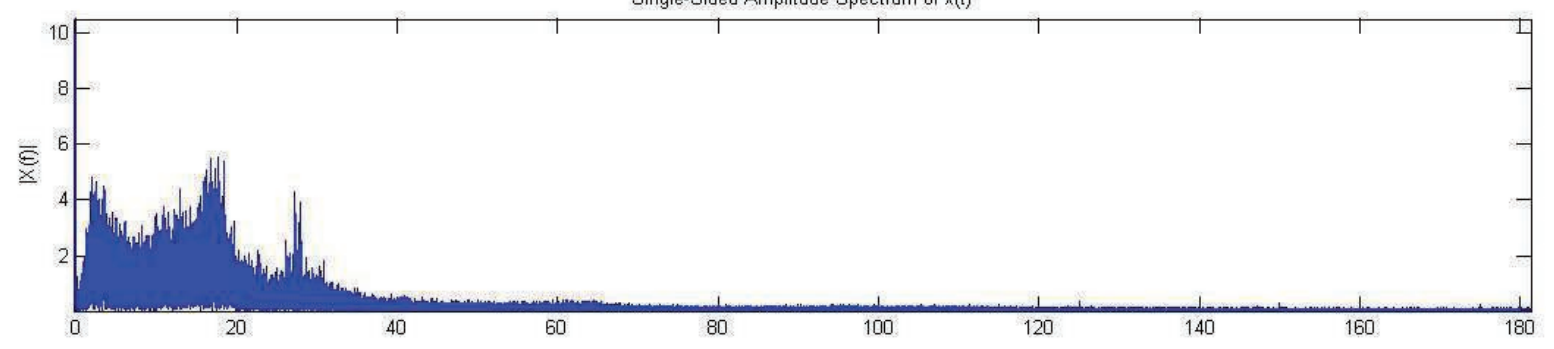

Picture 2: Vibration measurements (above, y-axis: milli-gs, $x$-axis: seconds) and frequency spectrum (in Hertz, below) of a vehicle

All the mentioned applications are not volume or cost limited. This means large board space for the energy harvesting power supply and medium or high investments are motivated by the application and potential savings. These are caused by the advantages of energy harvesting, like no wiring or installation expenses and early detection of malfunction, failure or down-time.

\section{Challenges of different ambient energy sources}

Depending on the chosen kind of ambient energy, different challenges are faced by the system designer. These can be handled at different points of the system or in different components like energy transducer, power management or energy storage.

Regarding thermal harvesters, their output voltage is proportional to the thermal gradient and the number of thermo-couples connected electrical in series and thermally in parallel. Furthermore, stateof-the-art electronics like wireless transceivers or sensors need a certain minimum voltage for proper operation. Thus, to increase the output voltage at low thermal gradients using large thermo-generators (TEGs) with a lot of elements is the simplest but not the most economic solution. Bearing in mind the high prices for thermo-electric materials, larger TEGs will results in higher system costs aside from larger board space. More sophisticated solutions, which enable higher miniaturization and lower system price use step-up voltage converters to boost the output voltage from small and cheap TEGs. One example is the Micro-Energy Power Management ASIC (ME-PMA2), which can work with voltages down to $20 \mathrm{mV}$ and can use minimum thermal gradients from a small number of thermocouples [3][4].

Another issue in energy harvesting is the efficiency of the power management, which is defined as the relation of output power to input power. A lot of power management circuits, mainly dc-dc converters exhibit a good efficiency only at a certain discrete power level and decrease their efficiency when they leave their optimum operation point. Especially in applications with a large dynamic range of the ambient energy in terms of thermal gradient, light intensity or vibration amplitude, a broad operation range of the power management is also important. This ensures that all the power is available for the application and not wasted in the power management. Picture 3 is showing the efficiency of a dc-dc converter for TEGs or solar cells with very broad operation range. 
Output Voltage $3.8 \mathrm{~V}$

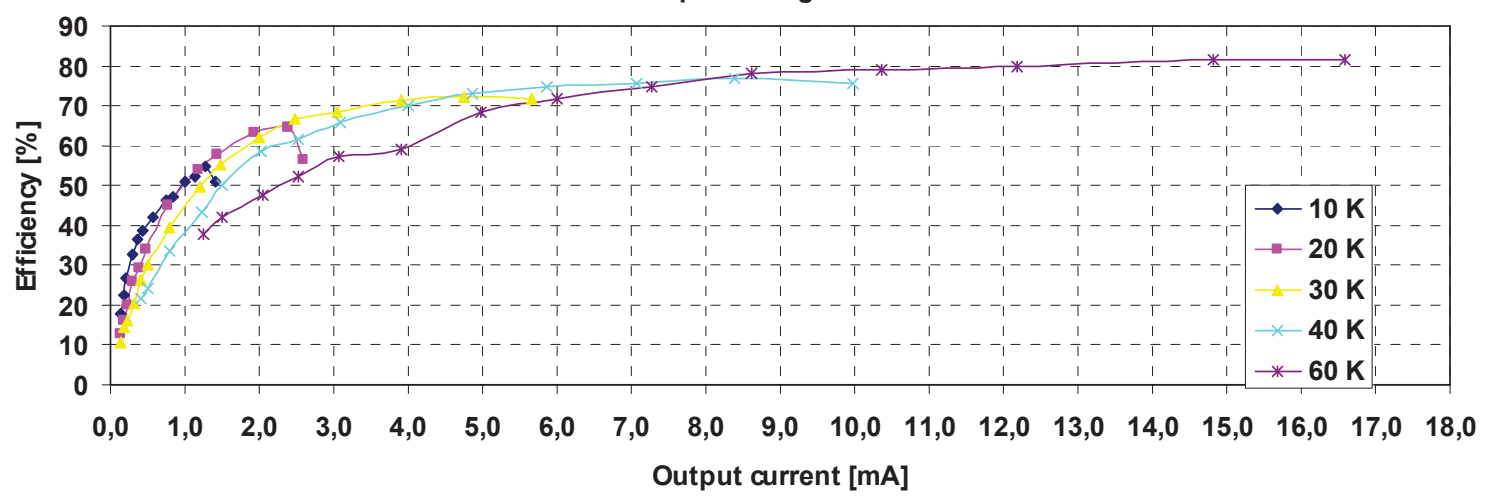

Picture 3: Efficiency of a dc-dc converter for energy harvesting with a broad operating range at different thermal gradients at a state-of-the-art TEG

Besides the dynamic range of the amplitude, the challenge in vibration energy harvesting is the frequency of the ambient energy source, like a motor, a pump or a moving vehicle. Most often, this exciting frequency is not constant. Adaptive vibration transducers, which control their resonant frequency according to the exciting frequency are difficult to design, since the control loop and control mechanism require additional power and degrade the efficiency of the whole system. Broadband transducers are easier to realize, but they exhibit a larger damping and thus a smaller efficiency over the whole frequency of operation. Applying several transducers and tuning them to different frequencies to achieve a broadband system with low damping ratio is a good approach when large device dimensions and system costs are acceptable.

Broadband operation is also an issue in the electronic power management. Aside from the standard method of rectifying and filtering the alternating current from the transducers, active switching ac-dc converters are superior. Picture 4 shows an SSHI (Synchronized switch harvesting on inductor) approach. A circuit implementation achieved efficiencies above $80 \%$, significantly higher than the standard approach (see Picture 5). By triggering the switching action with the input amplitude from the vibration transducers these converters are self-adapting to any input frequency in a limited bandwidth, e.g. $100 \mathrm{~Hz}$ and can work efficiently with broadband vibration transducers [5].

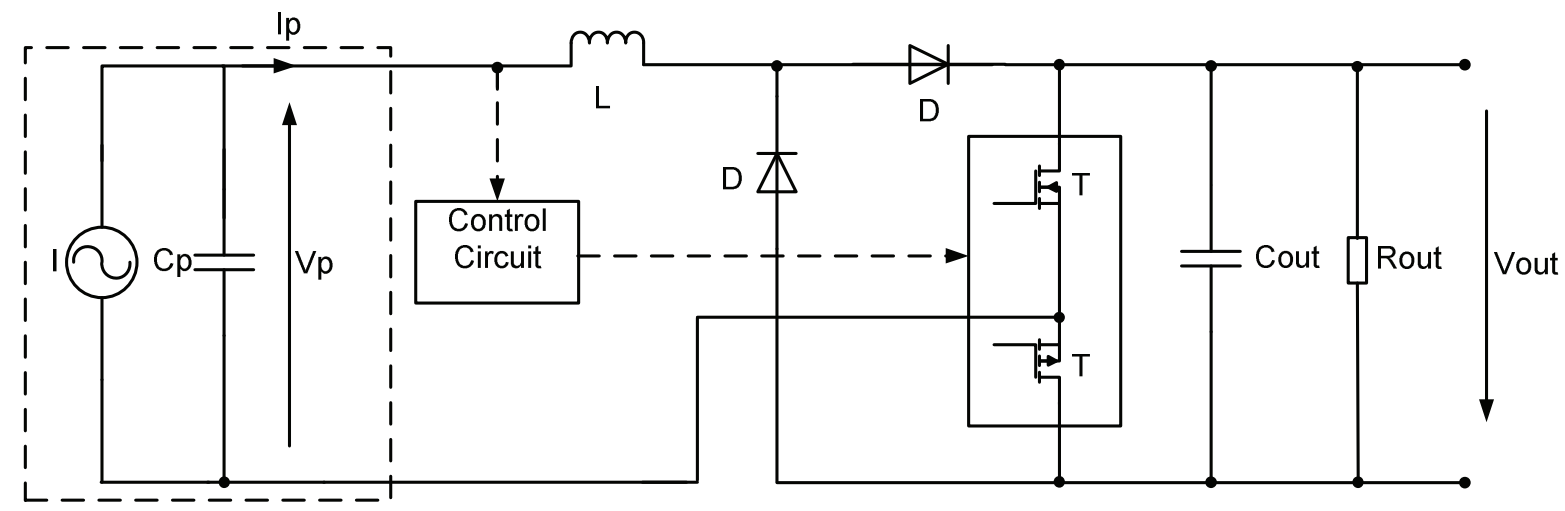

Picture 4: SSHI ac-dc converter for piezo-generators 


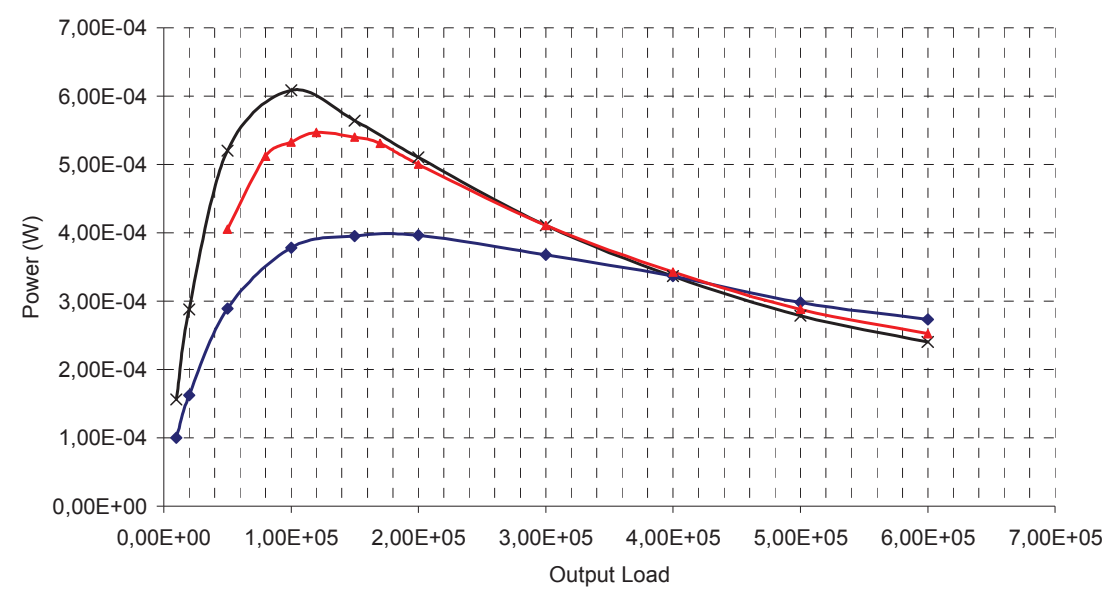

Picture 5: Comparison of different ac-dc converters for piezo-generators with equal input source (INVENT DuraAct with 17 Hz, 0.1 g; Blue: Standard; Red: SSHI; Black: Optimum load resistance)

\section{Summary and Outlook}

Energy Harvesting makes use of ambient energy to power small electronic devices. The typical system components like transducer, power management and energy storage have to be adapted to the energy source in use and the application requirements.

First successful applications are structural health monitoring and condition monitoring systems, where wireless sensor notes are powered from vibrations or thermal gradients. Limiting factors are often the costs or the size to achieve the required output power. Upcoming promising fields of operations will be the transport and logistics sector. Consumer applications or medical devices are predicted to become further markets for energy harvesting, whereas for these applications the price and the small output power are presently the parameters to optimize.

\section{References:}

[1] Roy Freeland: 'Laboratory Toy or Practical Power?', Symposium 'The Future of Energy Harvesting', IMEC, Leuven, 2010.

[2] Armin Anders: 'EnOcean - Integrated Approach for Energy Harvesting Wireless', IDTechEx Energy Harvesting Storage, Boston, 2010.

[3] Datasheet Micro Energy Power Management ASIC ME-PMA2.

[4] Jim Bierschenk: Optimized Thermoelectric Energy Harvesting Systems, IDTechEx Energy Harvesting Storage, Boston, 2010.

[5] I C Lien, Y C Shu, WJ Wu, S M Shiu and H C Lin: Revisit of series-SSHI with comparisons to other interfacing circuits in piezoelectric energy harvesting, Smart Material Structures 19 (2010) 125009

(12pp), IOP PUBLISHING. 